\title{
原始创新从哪里来：再谈化学中合成氨方法的发现
}

\author{
王亚琴 1,2 , 马腾 $2,{ }^{*}$ \\ 1 沈阳理工大学环境与化学工程学院, 沈阳 110159 \\ 2 沈阳农业大学理学院, 沈阳 110866
}

摘要: 从化学史上的合成氨技术着手, 探讨原始创新产生的全过程和影响因素, 并介绍科学家在合成氨研究中所经 历的一些曲折, 特别是哈伯(Fritz Haber)所做的贡献, 他发展的合成氨方法最终转化为人工固氮的主流技术, 对农业 和社会的发展产生了显著的影响。合成氨案例可以作为研究生树立原始创新的思想信念, 并为之不解奋斗的学习典 范; 也会对研究生的培养单位和指导教师有所启发。

关键词: 研究生培养; 原始创新; 合成氨; 化学热力学; 催化

中图分类号: G64; O6

\section{Revisiting Classic Cases of Original Discoveries and Inventions: Ammonia Synthesis in Chemistry}

\author{
Yaqin Wang ${ }^{1,2}$, Teng $\mathrm{Ma}^{2, *}$ \\ ${ }^{1}$ School of Environmental and Chemical Engineering, Shenyang Ligong University, Shenyang 110159, China. \\ ${ }^{2}$ Institute of Science, Shenyang Agricultural University, Shenyang 110866, China.
}

\begin{abstract}
The paper would revisit the classic story of ammonia synthesis and explore the untold details to get some hints about how an original idea or solution was produced in running research projects. There were generations of prominent scientists who had devoted to ammonia synthesis and failed with the loss of their fame, time or even lives. Fritz Haber accepted the challenge accidently, and confirmed a sound technological method that ammonia could be synthesized directly from its elements. Afterwards, his method was transferred successfully to the mainstream technology that influences the development of modern agriculture and society. The direct synthesis of ammonia has been used as one of the classic case studies of achieving original ideas or solutions by our graduate students in their professional careers. Furthermore, for the supervisors or service personnel in universities or research institutes, it would provide with an arsenal of spurring scientific discoveries or technological developments in graduate education programs.
\end{abstract}

Key Words: Graduate education program; Original discoveries and inventions; Ammonia synthesis;

Chemical thermodynamics; Catalysis

目前, 中国的快速发展已经到了非常关键的历史时期, 但是一些重要领域仍存在许多的瓶颈问 题, 对国家在科技方面的国际竞争力极为不利。这些瓶颈问题的根本在于基础研究薄弱, 具体表现 为缺少原始创新能力, 很多科研人员亦步亦趋地做研究, 既无法形成特色, 也不可能引领新的方向

收稿: 2020-08-12; 录用: 2020-09-30; 网络发表: 2020-11-16

“通讯作者, Email: mateng77@126.com

基金资助: 沈阳农业大学天柱山学者计划; 研究生教育教学改革研究项目(2019-yjs-36); 辽宁省教育厅科学研究经费项目(LSNJC201913) 
和未来趋势 ${ }^{[1]}$ 。研究生是中国科研事业重要的新生力量, 其创新创造能力正处于人生的黄金时期, 在一定程度上能体现出我国原始创新能力的水平。因此在研究生的培养过程中, 原始创新能力的培 养显得尤为重要, 它是高校教师的核心任务之一。

创新性的科研活动经常涉及到及时、巧妙地解决所面临的科学问题, 有时可能是发现全新的科 学问题。因此在实施科研项目的过程中, 研究生不仅需要采用新知识、新方法思考和解决问题; 还 要尽快地完成由知识的掌握者向创造者的新角色转换。研究生导师作为研究生科研活动的引路人, 肩负着 “言传、身教” 的责任, 既要积极示范、热情鼓励, 也得严格要求, 才能使研究生顺利地进 入新角色, 成为国家创新的栋梁之材 ${ }^{[2]}$ 。此外, 在研究生的学位课程中增加创新经典案例环节也是 很有必要的, 它的作用与研究生导师个人的科研经验相似, 但又有明显不同。

创新经典案例一般取材于科学发展史中里程碑式的研究成果, 因其支持材料较为丰富多样, 又 有典型性, 比较适合研究生通过分析、思考快速地获取科研工作 “经验” , 是对研究生导师 “言传、 身教” 过程的有益补充。在创新案例中出现的人物, 大多数是世界知名的科学家或发明家, 他们的 成功案例无疑会成为研究生在创新道路上独辟蹊径、奋勇前行、百折不挠的精神驱动力 ${ }^{[3]}$ 。笔者曾 经在 “环境催化化学” 等研究生课程中尝试引入一些经典的创新案例, 得到相关研究生和导师的认 可, 教学效果良好。本文选取了其中的一个课程案例, 即合成氨的研发背景和曲折历程, 详细地进 行阐述和探讨, 希望它能对研究生原始创新能力的培养有所帮助。

\section{1 十九世纪末的危机}

19世纪末, 人口的增长给社会带来新的危机：如何在有限的土地上解决粮食的供给问题？有些 社会学家认为, 只有通过战争或疾病才能解决人口过多的问题; 而化学家们给出的解决方案无疑是 更合适的。英国的克鲁克斯(William Crookes)提出, 通过化学方法把空气中的氮气转化为植物可吸收 的氮肥(氨盐或硝酸盐化合物), 能够促进粮食的高产 [4]。当时欧洲氮肥的主要来源是南美秘鲁、智利 的矿石资源和焦炭制造过程的副产物氨, 前者是不可再生的, 且储量有限, 而后者的产量较小, 两 者均无法满足农业的长远需求。

要实现把空气中的氮气转化为植物可吸收的氮肥, 首要的困难是如何把氮气分子内强的化学键 破坏, 释放出氮原子并与其他元素的原子相结合, 即氮元素的固定问题。当时已经发现两种方法, 一种是让氮气和氧气在高压电弧的激发下形成氮氧化物, 另一种是借助氮气与碳化钻之间的反应形 成氰氨化钙, 再与水蒸气反应即可形成氨气。这两种方法都有一个共同的缺点, 属于高能耗的过程, 运行成本较高。

因此, 人们开始思索其他可行的方法, 即由氮气和氢气直接进行化学反应, 一步合成氨气分子。 从18世纪末期开始, 多种实验方法和条件(高压、高温和不同的催化剂)均被尝试过, 但进展仍然比较 缓慢。到了19世纪末, 拉姆塞(William Ramsay)和杨(Sydney Young)研究氨气分解时发现, 氨在 $800{ }^{\circ} \mathrm{C}$ 无法实现完全分解, 总是有一小部分未分解的氨存在于反应器中 ${ }^{[5]}$ 。根据热力学中化学平衡的观点, 在同样的条件下氨气也可以由氮气和氢气直接合成。虽然拉姆塞和杨的合成氨实验最后也没有成功, 但是它重新燃起化学家在 20 世纪初对合成氨研究的兴趣。

\section{2 大师们的败走麦城}

合成氨研究的重大意义, 当时很多的化学家心里都比较清楚, 这包括德国的奥斯特瓦尔德 (Wilhelm Ostwald)、法国的勒沙特列(Henry Louis Le Châtelier)等多位大师级人物在内 ${ }^{[5]}$ 。

奥斯特瓦尔德在1900年声称, 他的实验室使用铁的催化剂使氮气和氢气在高温高压下反应, 成 功地合成出氨气并为此申请了专利。工业巨头巴斯夫(BASF)公司指派刚毕业不久的工程师博施(Carl Bosch)去做重复实验, 结果发现奥斯特瓦尔德的催化剂受到了污染, 含有氮化铁杂质。真正发生反 应的是氮化铁与氢气, 而不是氮气和氢气。奥斯特瓦尔德意识到自己的错误后, 撤回了专利, 不过 
专利的要点在后来开发成功的方法中基本上得到保留: 高温、高压、含铁的催化剂和气体的循环使 用。

勒沙特列同时也在进行合成氨的实验, 事前他对反应的温度、压力和铁催化剂等参数条件进行 了仔细的测算, 可是他的反应装置在实验中意外地发生爆炸, 损失惨重。事故发生后, 勒沙特列不 得不中止合成氨方面的研究。这也说明气体高压反应的实验技术尚未完全成熟, 仍存在一定的危险 性。

这些知名教授的先后失利, 让学术界和工业界对于合成氨是否具备可行性产生了怀疑。在这种 情况下, 合成氨研究的前景不再被看好, 所以一般人也不愿轻易地接触这个难题。

\section{3 一波三折的成功}

\section{1 哈伯的小项目}

1904年, 德国卡尔斯鲁厄工业大学(Technische Hochschule Karlsruhe)的副教授哈伯(Fritz Haber) 接到一家小公司(Margulies brothers, 由奥地利的马古里兄弟经营)的科研项目, 对方希望能对合成氨 的可行性进行探索。哈伯对奥斯特瓦尔德的合成氨实验早有耳闻, 他有些犹豫不决。哈伯出生于 1868 年, 大学期间在柏林、海德堡等地学习有机化学, 1891年获得博士学位(导师为Karl Liebermann教授)。 毕业之后, 他辗转到多家化工厂短期工作, 但不太如意。哈伯还曾两次计划到奥斯特瓦尔德的实验 室学习和工作, 均被婉言拒绝。1894年, 哈伯来到卡尔斯鲁厄工业大学, 跟随邦特(Hans Bunte)和恩 格勒(Karl Engler)两位教授从事烃类化合物(石油)的热分解和燃烧学研究 ${ }^{[6]}$ 。此后他如鱼得水、边干 边学, 开始在物理化学领域崭露头角。受到同事兼朋友鲁金(Hans Luggin)的影响, 哈伯还在电化学 领域小试牛刀, 取得了不错的成绩。1898年哈伯出版了一本电化学方面的著作《工业电化学》, 获到 同行的认可，同年他晋升为副教授。

最后, 他强烈的自尊心和爱冒险的个性占了上风, 同意开展合成氨的实验研究。虽然在合成氨 方面没有任何经验, 但哈伯对于气体反应的热力学和动力学研究早已是轻车熟路。这得益于卡尔斯 鲁厄学术研究的大环境, 以及哈伯自己十年来辛勤的工作。哈伯采取的办法是研究合成氨的逆反应, 即氨分解反应, 通过平衡时氨的浓度进行考查。可是实验结果不是太好, 哈伯将这个消息通报给马 古里兄弟后, 在1905年将实验数据整理成论文发表。然而, 该论文的发表给哈伯带来了大麻烦。

\section{2 为荣誉而战}

1906年奥斯特瓦尔德从莱比锡大学退休, 他的学生能斯特(Walther Nernst)教授成为物理化学的 领军人物。此时, 能斯特刚提出热力学第三定律, 他迫切地需要一切能对理论进行验证的反应数据。 能斯特找到了哈伯在1905年发表的数据, 并特意安排了一名学生进行重复实验, 结果与哈伯发表的 数据不一致。于是他给哈伯写信讨论这一情况。1906年对哈伯也很重要, 他如愿以偿地晋升为教授, 信心满满地准备开创一番事业。接到能斯特的来信后, 哈伯不敢急慢, 重新进行了实验, 发现新结 果的确与能斯特的结果比较接近。哈伯将新结果写信通报给能斯特, 它甚至比能斯特本人的结果还 要好一些。然而, 此事并没有结束。

1907年本生学会(Bunsen Society)的会议在德国汉堡召开, 哈伯遭到能斯特强烈的公开抨击, 对 方指责他的实验方法和结果极不可靠, 这对刚晋升为教授的哈伯来说是一个奇耻大辱 ${ }^{[7]}$ 。两者的学 术地位悬殊, 与能斯特的交恶将给哈伯的荣誉和职业发展带来极为不利的影响。会议结束后, 哈伯 一回到学校就病倒了, 不得不进行休养、治疗。

本生会议发生的意外事件打乱了哈伯的工作计划, 使合成氨研究成为他根本无法放下的课题。 哈伯的英国助手勒罗西尼奥尔(Robert Le Rossignol)重新设计实验装置 ${ }^{[8]}$, 对以往的合成氨实验进行 了重复和补充, 新的热力学数据让哈伯感觉到它甚至有工业应用的前景。此外, 哈伯也在积极尝试 其他的固氮方法, 比如使用电弧使氮气和氧气反应生成一氧化氮, 并率先实现了突破。经恩格勒的 大力举荐, BASF同意对哈伯的研究工作进行资助, 但合作仅限于电弧法固氮, 并不包括举世闻名的 
合成氨方法。与BASF进行合作的过程中, 哈伯一步步地向对方展示合成氨新方法的可行性和光明前 景, 在他解决催化剂和高压下的产物分离等一系列问题之后, 双方最终签订了新的开发合同。前面 提到的工程师博施负责合成氨方法的工业化放大试验, 他需要解决的技术问题主要有三个: 氢气的 经济获取、稳定价廉的催化剂和适用于生产的高压反应装置。1913年10月, BASF在奥帕(Oppau)的 新工厂完成建设后开始运行, 氨的产量约为 10 吨/天; 一年后氨的产量提高到 20 吨/天, 主要用于生产 氮肥硫酸铵 ${ }^{[9]}$ 。此后, 哈伯与博施的方法成为世界上主要的合成氨技术, 他们俩也分别于1918年和 1932年先后获得诺贝尔化学奖。

\section{4 关于合成氨的思考与启示}

1) 合成氨研究极具物理化学的特点: 合成氨反应条件的探索离不开化学热力学的指导和影响, 特别是压力和温度的选择; 反应需要使用催化剂加快反应速率, 减少达到平衡的时间。哈伯在物理 化学方面并没有接受过正规的训练, 但是他凭着在气体反应研究中积累的经验, 自觉地学习和运用 物理化学研究的新方法, 深入地思考且不惧权威的论断, 成功地解决了合成氨这个难题。这说明原 始创新需要有坚实的理论基础, 只有依赖先进的理论或者方法, 才能真正做到自信和有的放矢, 并 减少失败的机率。

因此在培养研究生的过程中, 要灵活地应用多种教学方式, 不断加强基础理论以及新思想(方法) 的学习。研究生处于大脑思维最活跃的时期, 基础理论和新思想接受程度高, 即使不能马上生效, 也可以受益终生, 从而成为原始创新的基石。所以, 一方面要在课程学习阶段聘请优秀教师授课 ${ }^{[10]}$, 另外也要在实验室经常召开组会, 讨论本领域内新的进展情况, 它们均是原始创新能力培养过程中 必不可少的环节。

2) 原始创新往往并非一蹴而就, 中间夹杂着许多意外因素, 这使得一些成果极具个人色彩。本 文中的哈伯就是在他个人的荣誉受到威胁时, 才激发出研究合成氨时的义无反顾, 反应条件的设定 也颇具冒险精神。也可以说, 能斯特的攻击激发出哈伯的创新潜能, 成就了后者在合所氨方面的突 破。当然卡尔斯鲁厄工业大学在气体反应方面厚重的传统和积累也发挥了较好的支撑作用, 这使得 哈伯进行气体高压实验时能有所凭借, 而非盲目的冒险。

研究生正处于情感丰富的青年时期, 个人情绪在受到外界环境的影响后, 难免会出现波动起伏 的情况。当个人情绪被控制在适当紧张的范围之内时, 对主观能动性和创新思维的激发非常有利。 因此分配研究生的科研工作时需要考虑其个性特点, 任务的难度和指导方式也要因人而异、适可而 止。对于有冒险精神的研究生, 适当安排一些具有挑战性的探索项目, 使其在压力面前增强主观能 动性, 充分挖掘其创新潜能。当然, 研究生个人的创新最好与研究组长期的积累密切相关, 只有这 样他才能在前进的道路中有所凭借, 减少学习的时间与机会成本, 较快地完成目标。

3) 在人工固氮方面, 哈伯不仅高度重视由氮气和氢气直接合成氨的研究路线, 还沿袭了高压电 弧法合成一氧化氮的研究路线。后者是哈伯1902年去美国考察时学习得到的, 当时在美洲刚开始有 示范性的应用, 这也许是他在欧洲获得资助的主要原因之一。电弧法合成一氧化氮的研究虽然没有 取得最终的成功, 但它使哈伯得到了比较充足的科研经费, 可以在一段时间内维持高风险的合成氨 研究, 并有时间和机会向外界宣传其最新的成果。

因此给研究生安排的工作量要适当, 使其在工作中能有时间关注本领域中相关研究方向的进展, 形成一定水准的学术品味和鉴赏能力。积极安排研究生参加国内外的学术会议等交流活动, 对研究 生拓宽学术视野、培养创新能力和取得学术成功也是大有禆益的。

4) 令人遗憾的是在第一次世界大战期间, BASF公司制造的氨主要服务于战争的需要, 即用于 生产炸药而不是氮肥。当氮肥在全世界的土地上广泛施用后, 人们还发现过量的施用不仅能引起土 壤质量劣化; 还在一定程度上促进了雾䨪天气的形成, 给人类的健康带来新的危害 ${ }^{[11]}$ 。这些现象表 明, 科学家的创新也许并不能完美地解决社会的危机问题, 它仍然需要社会其他成员不断增强的责 
任感与仁慈心进行配合，才能有效地减少负面的影响。

总之，合成氨方法的发现是现代化学史上的经典大作，即使在今天看来它仍然有很多好的启示， 需要我们不断地学习和领悟, 并应用到研究生(高年级本科生有时也适用)教育与原始创新能力培养 的过程中去, 提高青年创新人才的培养质量。

[1] 李克强. 国家杰出青年科学基金工作座谈会. [2019-09-04]. http://www.mot.gov.cn/guowuyuanxinxi/201909/t20190904_3246505.html

[2] 江元生. 化学进展, 2011, 23 (12), 2399.

[3] Jackman, J. A.; Cho, D. J.; Lee, J.; Chen, J. M.; Besenbacher, F. ; Bonnell, D. A.; Hersam, M. C.; Weiss, P. S.; Cho, N. J. ACS Nano 2016, 10 (6), 5595.

[4] Charles, D. Master Mind: The Rise and Fall of Fritz Haber, the Nobel Laureate Who Launched the Age of Chemical Warfare; HarperCollins: New York, USA, 2007: pp. 73-95.

[5] Smil, V. Enriching the Earth: Fritz Haber, Carl Bosch, and the Transformation of World Food Production; MIT Press: Cambridge, UK, 2004; pp. 61-82.

[6] Haber, F. Aus Leben und Beruf; Verlag von Julius Springer: Berlin, Germany, 1927; pp. 1-6.

[7] Schindewolf, U. Bunsen-Magazin 2000, 2 (6), 138.

[8] Sheppard, D. Notes Rec. R Soc. Lond. 2017, 71 (3), 263.

[9] Coffey, P. Cathedrals of Science: The Personalities and Rivalries That Made Modern Chemistry; Oxford University Press: Oxford, UK, 2008; pp. $71-94$.

[10] 顾日良, 陈范骏, 张俊伶, 袁力行, 邹春琴, 陈新平, 申建波, 江荣风, 张福锁. 学位与研究生教育, 2013, No. 7, 42.

[11] Wu, Y. Y.; Gu, B. J.; Erisman, J. W.; Reis, S.; Fang, Y. Y.; Lu, X. H.; Zhang, X. M. Environ. Pollut. 2016, $218,8$. 